\title{
Elevated TrkA receptor expression is associated with all-trans retinoic acid-induced neuroblastoma differentiation
}

\author{
Q. Gao ${ }^{1 *}$, C.F. Chen ${ }^{2 *}$, Q. Dong ${ }^{3}$, L. Hou ${ }^{4}$, X. Chen ${ }^{3}$, Y.L. Zhi ${ }^{3}$, X. Li ${ }^{3}$, H.T. Lü ${ }^{3}$ and \\ H.Y. Zhang ${ }^{3}$ \\ ${ }^{1}$ Department of Pediatric Surgery, Qingdao Women and Children Hospital, \\ Qingdao, Shandong, China \\ 2Department of Pediatric Surgery, Linyi People's Hospital, No. 27, Jiefang Road, \\ Linyi, Shandong, China \\ ${ }^{3}$ Department of Pediatric Surgery, The Affiliated Hospital of Qingdao University, \\ Qingdao, Shandong, China \\ ${ }^{4}$ Department of Biochemistry, Medical College, Qingdao University, Qingdao, \\ Shandong, China \\ ${ }^{*}$ These authors contributed equally to this study. \\ Corresponding author: H.T. Lu \\ E-mail: leihanleihan@126.com \\ Genet. Mol. Res. 14 (4): 13195-13202 (2015) \\ Received April 15, 2015 \\ Accepted August 19, 2015 \\ Published October 26, 2015 \\ DOI http://dx.doi.org/10.4238/2015.October.26.15
}

\begin{abstract}
Neuroblastoma is the most common and one of the deadliest among pediatric tumors; however, a subset of infants with neuroblastoma display spontaneous regression. The mechanism of spontaneous regression remains to be elucidated. TrkA plays an essential role in the differentiation and functionality of neurons; abundant TrkA expression is associated with favorable prognosis of neuroblastoma. All-trans retinoic acid (ATRA), a first-line drug for acute promyelocytic leukemia (APL) treatment, has been shown to induce differentiation and inhibit cell growth. Neuroblastoma tissues in our hospital inpatient were collected, primary cell culture was performed, and the cells were separated and purified to be
\end{abstract}


cell line. Trypan blue exclusion was used to count the numbers of cells alive, morphological changes were observed under the phase-contrast microscope. RT-PCR was used to determine the expression level of TrkA. In this study, a human neuroblastoma cell line was successfully established; in addition, we demonstrated that ATRA induces growth arrest and promotes the differentiation of neuroblastoma cells. In addition, ATRA was shown to significantly increase the levels of TrkA mRNA expression. Therefore, we concluded that the elevated expression of the TrkA receptor is associated with ATRA-induced growth arrest and differentiation o neuroblastoma cells. The results of this study provide a theoretical basis for the clinical application of differentiation-inducing ATRA for neuroblastoma therapy.

Key words: Neuroblastoma; Differentiation; TrkA; All-trans retinoic acid

\section{INTRODUCTION}

Neuroblastoma (NB) is one of the most common pediatric neoplasms, originating from immature neuroblasts in the peripheral nervous system (Brodeur et al., 2009; Cheung and Dyer 2013). NB shows remarkable heterogeneity, ranging from a favorable outcome because of spontaneous regression or complete remission after minimal therapy, to an unfavorable outcome due to relentless progression in spite of intensive multimodality therapy (Brodeur et al., 2009). This may be attributed to its heterogeneity at a molecular level, suggesting that the development of NB may involve a block in the differentiation of neural crest precursor cells. Recent studies have demonstrated that the TRK family of neurotrophin receptors plays an important role in the diverse behavior of NB (Chang et al., 1998; Brodeur et al., 2009).

TrkA, a principal receptor of nerve growth factor (NGF), belongs to the TRK family of neurotrophin receptors, and displays the characteristic features of tyrosine kinase (TK) receptors (Snider, 1994). The binding of NGF to TrkA promotes the growth, differentiation, and survival of neural cells (Kaplan and Miller, 2000). The TrkA receptor undergoes dimerization, followed by autophosphorylation regulated by the intrinsic receptor tyrosine kinase, upon interaction with NGF (Jing et al., 1992). The activated receptor complex mediates the signal via the ras, raf, and MEK pathways to the nucleus, where the immediate response genes are activated (van der Geer et al., 1994). Previous reports have revealed that TrkA regulates the growth and differentiation of NB cells in vitro and in vivo (Matsushima and Bogenmann, 1993; Kim et al., 2002). TrkA has been reported to play a pivotal role in NGF-induced differentiation of in vitro cultured neurogenic cells, including NB cells (Sugimoto et al., 2001). TrkA expression is also important for the development of normal sympathetic neurons, and current evidence has demonstrated that TrkA expression is greatly associated with a favorable prognosis of NB; in fact, low or absent TrkA expression is correlated with poor outcome.

Retinoic acid (RA), the biologically active derivative of vitamin $A$, has three isomers: all-trans RA (ATRA), 9-cis RA, and 13-cis RA. Both neurotrophins and RA play important roles in developmental neurogenesis, involving differentiation, death, and survival of neurons (Siddikuzzaman et al., 2011). ATRA induces morphological and molecular changes in human NB, including responsiveness to neurotrophins. ATRA is the biological ligand for the retinoic acid receptor (RAR), and is one of the most potent in vitro inducers of human neuroblastoma 
differentiation (Heyman et al., 1992).

Spontaneous regression has been found in many types of human cancers; the greatest number of cases of spontaneous regression have been documented in patients with NB (Evans et al., 1976; Papac, 1998). A subset of NB patients experience regression after minimal or no treatment. The mechanism of regression has become the focus of recent studies investigating novel strategies for the therapy of human cancers. A large number of reports suggest that the NGFTrkA pathway contributes to the spontaneous regression of NB, and that abundant TrkA expression causes growth arrest and promotes the differentiation of NB cells into a regressed or benign state (Chang et al., 1998; Brodeur et al., 2009). In this study, we observed ATRA-induced growth arrest and differentiation associated with higher TrkA mRNA expression in human NB cells, with an aim of providing a theoretical basis for the clinical application of differentiation-inducing reagents for NB therapy; in addition, we attempted to determine the possible mechanism of spontaneous regression of NB.

\section{MATERIAL AND METHODS}

\section{Establishment of NB cell line and cell culture}

The human NB cell line was established from a 2-year old NB patient from our hospital. The cells were cultured in Dulbecco's modified Eagle's medium (DMEM), containing 10\% fetal bovine serum (FBS; Invitrogen, Carlsbad, CA, USA), penicillin (100 U/mL), and streptomycin $(0.1 \mathrm{mg} / \mathrm{mL})$. The cells were incubated at $37^{\circ} \mathrm{C}$ in a humidified incubator, containing $5 \% \mathrm{CO}_{2}$ (Chang et al., 1998).

\section{Cell treatment}

The NB cells were divided into three groups: in group $A$, the cells were exposed to 5 $\mu \mathrm{mol} / \mathrm{L}$ of ATRA; the cells of group B were exposed to $20 \mu \mathrm{mol} / \mathrm{L}$ of ATRA, and group $\mathrm{C}$ was the control group, where cells were cultured in DMEM without ATRA. Each group was prepared in quadruplicate.

\section{Viable cell count}

Cells $\left(2 \times 10^{5}\right)$ were seeded into the wells of a 24-well culture plate containing $1.5 \mathrm{~mL}$ culture medium/well. The cells were treated with or without ATRA, as described above. The viable cell count was determined $0,2,4,6$, and 8 days after ATRA treatment, using the trypan blue exclusion method.

\section{Analysis of cell differentiation}

The cells were seeded at a density of $1 \times 10^{3}$ cells $/ \mathrm{cm}^{3}$ in $25 \mathrm{~cm}^{2}$ culture flasks and treated with or without ARKA, as described in a previous subsection. The cell differentiation was analyzed $0,2,4,6$, and 8 days after treatment by the Sandquist method, as described in a previous study (Kim et al., 1999): the axons of the differentiated cell must extend to more than twice the diameter of the cell body. The rate of cell differentiation was determined based on five views of 200 cells each. 


\section{Reverse transcription polymerase chain reaction $\mathrm{RT}-\mathrm{PCR}$}

The total RNA was extracted from the NB cells of each group using the TRIzol reagent (Invitrogen) according to the manufacturer protocols. The total RNA was reverse transcribed using TaqMan reverse transcription reagent (Applied Biosystems, Foster City, CA, USA), and the newly generated cDNA was amplified using the Gene Taq PCR reagent (TaKaRa Bio Inc., Otsu, Japan) following the manufacturer protocols. The reaction conditions were set as follows: one cycle of denaturation at $94^{\circ} \mathrm{C}$ for $4 \mathrm{~min}, 35$ cycles each of denaturation at $94^{\circ} \mathrm{C}$ for $30 \mathrm{~s}$, annealing at $60^{\circ} \mathrm{C}$ for $30 \mathrm{~s}$, and extension at $72^{\circ} \mathrm{C}$ for $1 \mathrm{~min}$, and a final extension at $72^{\circ} \mathrm{C}$ for $10 \mathrm{~min}$. The primers used for the PCR amplification of TrkA were as follows: 5'-CTGGGCGGAGTGCCTGAA-3' and 5'-GGCTCCGGCTCCAGGAA-3'. An appropriate volume $(10 \mu \mathrm{L})$ of each PCR product was electrophoresed on a $2.5 \%$ agarose gel, and visualized with ethidium bromide (EB). The expression of TrkA mRNA was normalized to that of the housekeeping gene GAPDH. The amplified TrkA fragment was $528 \mathrm{bp}$ long, while that of GAPDH was $400 \mathrm{bp}$.

\section{Statistical analysis}

All data was analyzed using SPSS (v.12.0) software (IBM, Armonk, NY, USA), and results presented as mean \pm standard deviation (S.D.). Student's $t$-test was used to assess the statistical differences between two groups. $P$ values $<0.05$ were considered to be statistically significant.

\section{RESULTS}

\section{ATRA induces growth arrest in NB cells}

We observed a significant increase in the rate of inhibition of cell proliferation after treatment with ATRA. Table 1 indicated that the number of cells was similar on the first 2 days. However, the number of cells remained significantly higher in group $C$ than those in groups $A$ and $B$ after 4,6 , and 8 days $(P<0.05)$; also, group $A$ has a greater number of cells than group $B(P<$ 0.05). Cells in group $C$ showed normal proliferation, while the ATRA intervention notably inhibited the cell growth in groups A and B (Table 1). The proliferative ability of NB cells in group B (treated with $20 \mu \mathrm{mol} / \mathrm{L}$ of ATRA) was significantly attenuated, which was much more evident than that in group $A$ (treated with $5 \mu \mathrm{mol} / L$ of ATRA; $P<0.05$ ).

\begin{tabular}{|c|c|c|c|}
\hline \multirow[t]{2}{*}{ Time (day) } & \multicolumn{3}{|c|}{ Cell number $\left(10^{5} \mathrm{X}\right)$} \\
\hline & Group A & Group B & Group C \\
\hline 0 & $1.00 \pm 0.00$ & $1.00 \pm 0.00$ & $1.00 \pm 0.00$ \\
\hline 2 & $2.53 \pm 0.06$ & $1.25 \pm 0.04$ & $2.94 \pm 0.05$ \\
\hline 4 & $3.68 \pm 0.06^{\Delta}$ & $1.28 \pm 0.06^{\Delta}$ & $5.23 \pm 0.06$ \\
\hline 6 & $5.23 \pm 0.06^{\Delta}$ & $1.26 \pm 0.05^{\Delta *}$ & $7.56 \pm 0.13$ \\
\hline 8 & $6.16 \pm 0.04^{\Delta}$ & $1.15 \pm 0.11^{\Delta *}$ & $8.04 \pm 0.09$ \\
\hline
\end{tabular}

Compared to Group C, ${ }^{\Delta} \mathrm{P}<0.05$. Compared to Group A, ${ }^{*} \mathrm{P}<0.05$. 


\section{ATRA promotes differentiation of NB cells}

The NB cells in groups A and B showed remarkable morphological differentiation, including neurite extension, after ATRA treatment (Figure 1; Table 2). Neurite extension was apparent in groups $A$ and $B$ after ATRA treatment, and became longer and branched after 2 days (Figure 1). However, the NB cells in Group $C$ showed no apparent morphological differentiation (Figure 1A). The percentage of cell differentiation at different times after treatment, and under the influence of different concentrations of ATRA is summarized in Table 2 . The percentage of cell differentiation increased significantly after 2 days in groups $A$ and $B$, compared to that seen in group $C(P<0.05)$. The percentage of cell differentiation increased gradually with the treatment period; in addition, the percentage of cell differentiation in group $B$ was always higher than that in group $A$ at the same experiential time $(P<0.05)$
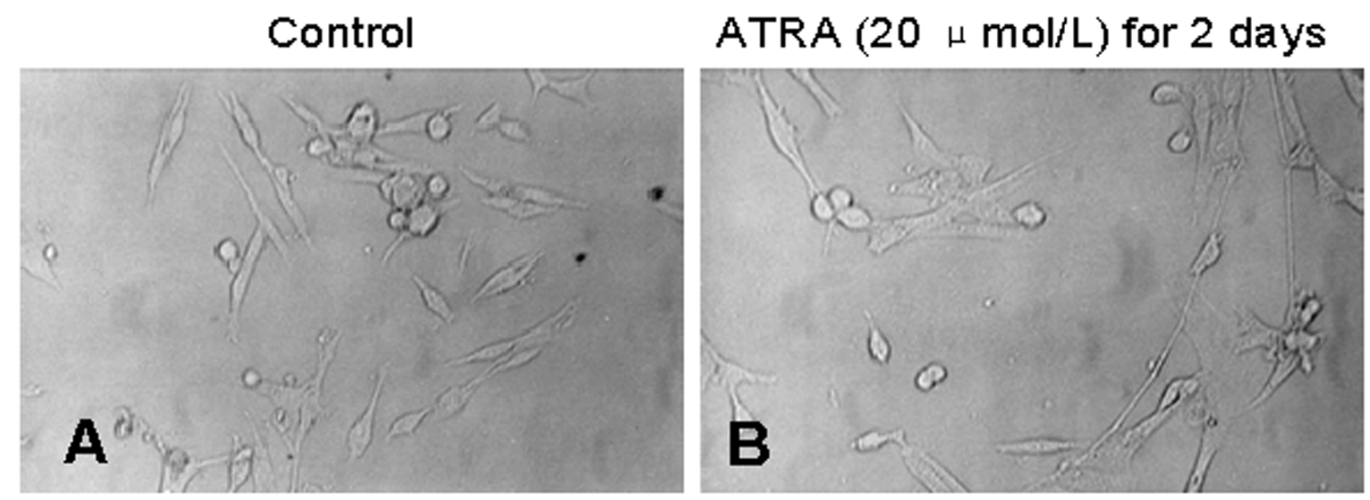

Figure 1. Microscopic observation of the morphological differentiation of NB cells (100X). NB cells were treated (A) without or (B) with $20 \mu \mathrm{mol} / \mathrm{L}$ of ATRA for 2 days.

Table 2. comparison of differentiation rates in different groups (means $\pm s$ ).

\begin{tabular}{lccr}
\hline Time (day) & \multicolumn{2}{c}{ Percentage of cell differentiation (\%) } \\
\cline { 2 - 4 } & Group A & Group B & Group C \\
\hline 0 & $5.80 \pm 0.57$ & $6.20 \pm 0.27$ & $5.50 \pm 0.50$ \\
2 & $19.20 \pm 0.84^{\Delta}$ & $33.40 \pm 1.14^{\Delta *}$ & $7.10 \pm 0.42$ \\
4 & $31.20 \pm 1.30^{\Delta}$ & $61.40 \pm 2.30^{\Delta *}$ & $8.20 \pm 0.57$ \\
6 & $32.00 \pm 1.58^{\Delta}$ & $67.40 \pm 2.31^{\Delta *}$ & $8.50 \pm 0.50$ \\
8 & $35.10 \pm 0.74^{\Delta}$ & $70.20 \pm 2.77^{\Delta *}$ & $8.90 \pm 0.74$ \\
\hline
\end{tabular}

Compared to Group $\mathrm{C},{ }^{\Delta} \mathrm{P}<0.05$. Compared to Group A, ${ }^{*} \mathrm{P}<0.05$.

\section{Expression of TrkA mRNA was elevated in NB cells treated with ATRA}

The level of expression of TrkA was higher in the groups treated with ATRA ( $A$ and $B)$ than that in group $C$ (no ATRA intervention; Figure 2). The TrkA levels increased notably in groups $A$ and $B 2$ days after ATRA intervention, compared to that in group $C(P<0.05$; Figures $2 A$ and $2 B)$. The TrkA mRNA expression was much higher in group B $(20 \mu \mathrm{M}$ ATRA) than that in group $A$ (treated with $5 \mu \mathrm{M}$ ATRA) 6 days after ATRA intervention ( $P<0.01$; Figures $2 \mathrm{C}$ and 2D). ATRA significantly elevated the TrkA mRNA expression in groups $A$ and $B$, compared to that seen in group $C(P<$ 0.01 ; Figures $2 \mathrm{C}$ and 2D). 


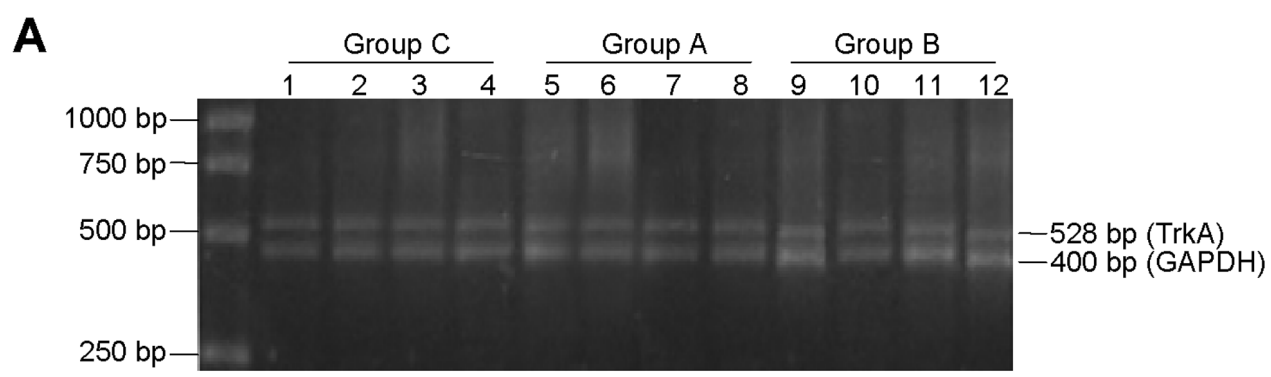

B
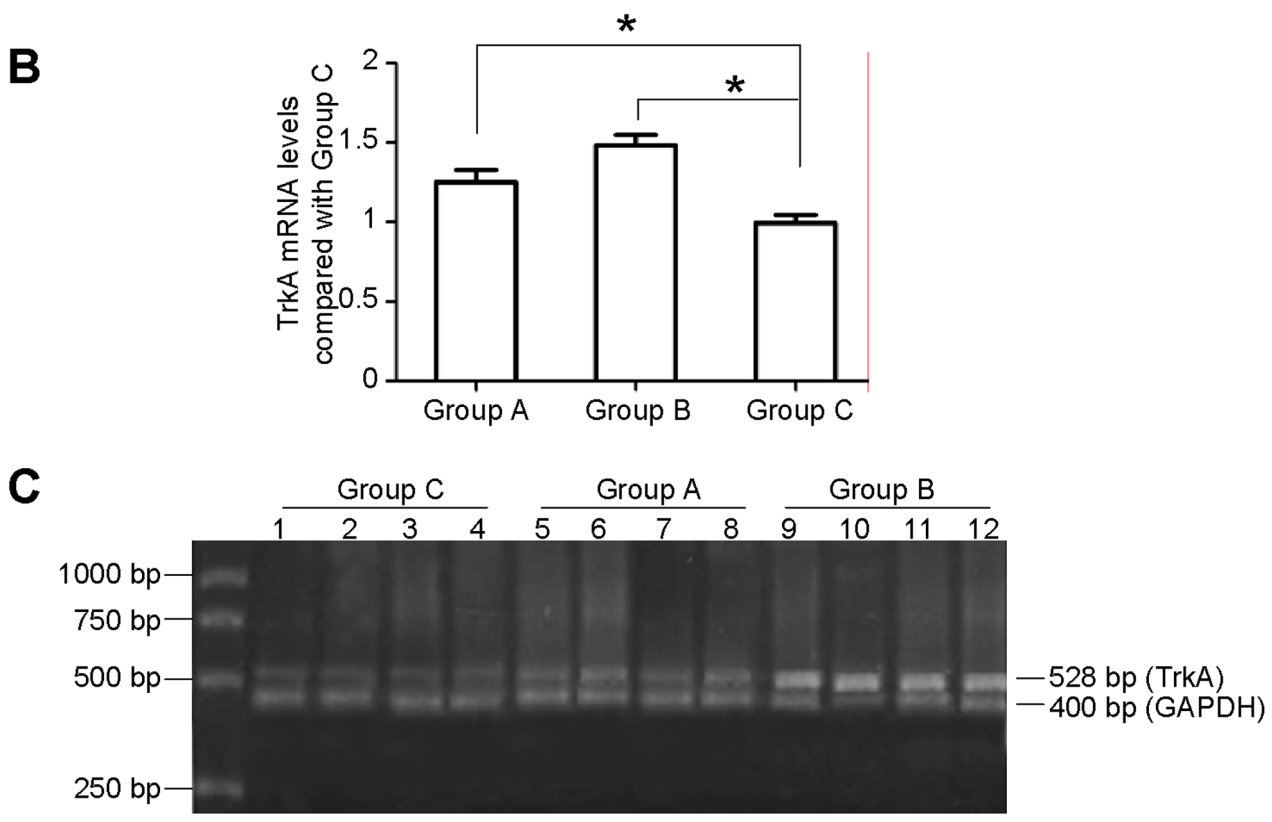

D

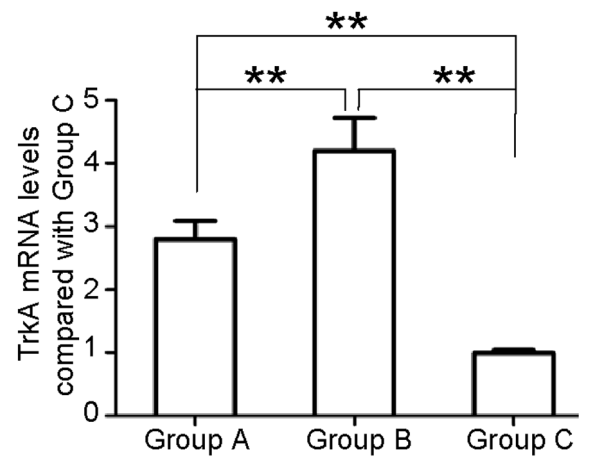

Figure 2. Analysis of TrkA mRNA expression. A. RT-PCR products of TrkA mRNA in different groups treated with or without ATRA for 2 days were analyzed by gel electrophoresis. B. Statistical analysis of TrkA mRNA levels in the different groups summarized in A. C. RT-PCR products of TrkA mRNA in the different groups (treated with or without ATRA for 6 days) were analyzed by gel electrophoresis. D. Statistical analysis of TrkA mRNA levels in the different groups summarized in $\mathrm{C}$. 


\section{DISCUSSION}

ATRA is a useful pharmacological reagent for chemoprevention and differentiation therapy of some human cancers (Masetti et al., 2012). ATRA has become the first choice drug for acute promyelocytic leukemia (APL) therapy because of its particularly impressive outcome in its treatment (Masetti et al., 2012). Previous studies have suggested that ATRA exerts an anti-tumor effect by regulating the cell growth and differentiation; therefore, in this study, we attempted to determine if ATRA inhibits NB cell growth and promotes NB cell differentiation into a regressed or benign state. The results showed that NB cells undergo differentiation after 2 days of treatment with ATRA ( $5 \mu \mathrm{mol} / \mathrm{L})$; this was characterized by a decrease in cell proliferation and morphological changes such as neurite extension. Many NB cells exposed to physiological concentrations of ATRA differentiated to a neuronal phenotype, and the proportion of differentiated cells increased with the ATRA treatment period. The results of this study also indicated that ATRA obviously affected NB cell growth arrest, and the proliferation ability was directly correlated with the concentration of ATRA. Based on these results, we concluded that ATRA induces growth arrest and promotes the differentiation of NB cells. In this study, a NB cell line was successfully established from fresh human NB specimens, which provides an excellent cell model system for ATRA-induced cell differentiation. ATRA induces the differentiation and inhibits the proliferation of NB cells; this suggested that ATRA may be used an anti-tumor therapeutic agent for differentiation therapy of NB, similar to APL therapy.

ATRA regulates cell growth and differentiation by modulating the gene expression, predominantly mediated by the ATRA-nuclear retinoic acid receptors (RARs) pathway (Masetti et al., 2012). TrkA has been shown to regulate the growth and differentiation of NB cells; TrkA expression plays a decisive role in the prognosis of NB, and is closely associated with spontaneous regression. Therefore, the role of TrkA in regulating the differentiation of NB cells exposed to ATRA, and the role of the ATRA-RAR pathway in the regulation of gene expression must be studied. In this study, ATRA was shown to upregulate the TrkA expression in NB cells. The mRNA level of TrkA increased and the morphological variation in NB cells also appeared after 2 days of ATRA treatment, whereas, the obvious growth arrest was observed after 4 and 6 days of ATRA treatment. The results indicated that the increase in TrkA expression precedes the growth arrest and morphological differentiation; that is, growth arrest and morphological variation were associated in part with the increase in TrkA expression. In other words, the NGF-TrkA pathway mediated the NB cell growth- and differentiation-regulating effects of ATRA. Therefore, TrkA may be one of the targeted genes modulated by the ATRA-RAR pathway during ATRA induction. However, the detailed signaling pathway and mechanisms remain to be elucidated.

This study revealed that ATRA induced growth arrest in and promoted the differentiation of NB cells, possibly by up-regulating TrkA mRNA expression at the transcriptional level. The elevated TrkA expression can be considered as a useful marker of favorable prognosis of NB; in addition, this is closely associated with the regressive state of NB. Therefore, we strongly propose that ATRA can be included in the development of an effective anti-tumor differentiation therapeutic strategy for NB.

\section{Conflicts of interest}

The authors declare no conflict of interest. 


\section{ACKNOWLEDGMENTS}

Research supported by grants provided by the Natural Science Foundation of China (\#NSFC 81272986 to Qian Dong and \#NSFC 81472542 to Lin Hou).

\section{REFERENCES}

Brodeur GM, Minturn JE, Ho R, Simpson AM, et al. (2009). Trk receptor expression and inhibition in neuroblastomas. Clin. Cancer Res. 15: 3244-3250.

Chang BB, Persengiev SP, de Diego JG, Sacristan MP, et al. (1998). Proximal promoter sequences mediate cell-specific and elevated expression of the favorable prognosis marker TrkA in human neuroblastoma cells. J. Biol. Chem. 273: 39-44.

Cheung NK and Dyer MA (2013). Neuroblastoma: developmental biology, cancer genomics and immunotherapy. Nat. Rev. 13: 397-411.

Evans AE, Gerson J and Schnaufer L (1976). Spontaneous regression of neuroblastoma. Natl. Cancer Inst. Monogr. $44: 49-54$. Heyman RA, Mangelsdorf DJ, Dyck JA, Stein RB, et al. (1992). 9-cis retinoic acid is a high affinity ligand for the retinoid X receptor. Cell 68: 397-406.

Jing S, Tapley P and Barbacid M (1992). Nerve growth factor mediates signal transduction through trk homodimer receptors. Neuron 9: 1067-1079.

Kaplan DR and Miller FD (2000). Neurotrophin signal transduction in the nervous system. Curr. Opin. Neurobiol. 10: $381-391$.

Kim CJ, Matsuo T, Lee KH and Thiele CJ (1999). Up-regulation of insulin-like growth factor-II expression is a feature of TrkA but not TrkB activation in SH-SY5Y neuroblastoma cells. Am. J. Pathol. 155: 1661-1670.

Kim GJ, Kim CJ, Cho SY, Chung IP, et al. (2002). Activation of trkA induces differentiation and inhibits the growth of JK-GMS Askin tumor cells. Lab. Invest. 82: 221-229.

Masetti R, Vendemini F, Zama D, Biagi C, et al. (2012). All-trans retinoic acid in the treatment of pediatric acute promyelocytic leukemia. Expert Rev. Anticancer Ther. 12: 1191-1204.

Matsushima $\mathrm{H}$ and Bogenmann $\mathrm{E}$ (1993). Expression of trkA cDNA in neuroblastomas mediates differentiation in vitro and in vivo. Mol. Cell. Biol. 13: 7447-7456.

Papac RJ (1998). Spontaneous regression of cancer: possible mechanisms. In Vivo 12: 571-578.

Siddikuzzaman, Guruvayoorappan C and Berlin Grace VM (2011). All trans retinoic acid and cancer. Immunopharmacol. Immunotoxicol. 33: 241-249.

Snider WD (1994). Functions of the neurotrophins during nervous system development: what the knockouts are teaching us. Cell $77: 627-638$

Sugimoto T, Kuroda H, Horii Y, Moritake H, et al. (2001). Signal transduction pathways through TRK-A and TRK-B receptors in human neuroblastoma cells. Jpn. J. Cancer Res. 92: 152-160.

van der Geer P, Hunter T and Lindberg RA (1994). Receptor protein-tyrosine kinases and their signal transduction pathways. Annu. Rev. Cell Biol. 10: 251-337. 